\title{
Oligonucleotides: Extrapolating from in vitro to in vivo
}

To the editor - In the field of therapeutic oligonucleotides, extrapolation of results acquired in vitro to the in vivo situation requires caution. The recent paper by Benimetskaya et al. ' provides compelling data indicating that the calcium-dependent integrin Mac-1 contributes to binding, incorporation and co-stimulatory action of oligonucleotides in granulocytes. We would like to stress three points: First, it has been shown by the authors that fibrinogen competes for cellular binding sites of oligonucleotides. Therefore, oligonucleotide binding and incorporation in the presence of fibrinogen in vivo may not be predicted by in vitro studies performed in the presence of fibrinogen-free serum. Secondly, the authors show that binding of phosphodiester but not of phosphorothioate oligonucleotides to the calcium-dependent integrin Mac-1 is dependent on extracellular calcium. However, binding was examined in the absence of molecules competing for the binding site on Mac-1, such as fibrinogen. In the presence of such molecules, binding of phosphorothioate oligonucleotide to Mac-1 may require assembly of binding sites facilitated by calcium. We have found that phosphorothioate oligonucleotide uptake into granulocytes in whole blood, their physiologic milieu, is strongly dependent on the presence of extracellular calcium (Hartmann, G. et al. Spontaneous oligonucleotide uptake in whole blood is dependent on physiologic extracellular calcium. Nature Biotechnology Antisense 97 conference, Cambridge, Mass., abstract 37). Finally, the authors demonstrated that in granulocytes, phosphorothioate oligonucleotides enhance TNF-stimulated generation of reactive oxygen species. By analogy, we found that in monocytes, phosphorothioate oligonucleotides enhance lipopolysaccharidestimulated TNF synthesis ${ }^{2}$. Since Mac-1 is expressed on the surface of both granulocytes and monocytes, oligonucleotide binding to Mac-1 might be responsible for co-activation of both cell species. In vivo, these co-stimulatory actions of phosphorothioate oligonucleotides on distinct leukocyte subpopulations may contribute to inflammatory reactions to local administration of these compounds. Such side effects have been observed in recent antisense studies for intravitreal (Kisner, D.L. Phase development of ISIS 2922 (Fomivirsen). Nature Biotechnology Antisense 97 conference) and subcutaneous ${ }^{3}$ injection.

\section{GUNTHER HARTMANN,}

MARTIN BIDLINGMAIER, BERND JAHRSDÖRFER \& STEFAN ENDRES Department of Clinical Pharmacology, Medizinische Klinik, Klinikum Innenstadt of the Ludwig-Maximilians-University, Ziemssenstr. 1, 80336 Munich, Germany

e-mail: ghartmann@lrz.uni-muenchen.de

Benimetskaya et al. reply - We thank Hartmann and colleagues for their comments. We do not disagree with their first point, but would like to point out that phosphorothioate oligodeoxynucleotide binding to Mac-1 is not an all-or-nothing phenomenon, even in the presence of competing fibrinogen. As usual, complex rate and equilibrium issues will predomi- nate in vivo. These will be governed by diverse factors, including oligonucleotide length, concentration and to some extent, sequence. With respect to point 2 , we have not claimed that the oligodeoxynucleotide uptake process, which is quite complex, cannot be calcium-dependent. However, in our hands, admittedly in vitro, the binding of phosphorothioate oligodeoxynucleotides to Mac-1 is calcium-independent. Note, however, that Mac-1 is not the only cell surface receptor for oligomers on neutrophils. It may account for only 40-50\% of total binding, depending on the state of neutrophil activation. Finally, it is of interest that stimulation of TNF- $\alpha$ synthesis in neutrophils after treatment with phosphorothioate oligodeoxynucleotides has been observed. Indeed, as Hartmann et al. suggest, it may be true that activation of Mac-1 by phosphorothioate oligodeoxynucleotides contributed to the "inflammatory reactions .....(that) have been observed in recent antisense studies for intravitreal and subcutaneous injection", but who's extrapolating now?

LYUBA BENIMETSKAYA, JOHN LOIKE \& C.A. STEIN

1. Benimetskaya, L. et al. Mac-1 (CD $11 \mathrm{~b} / \mathrm{CD} 18)$ is an oligodeoxynucleotide-binding protein. Nature Medicine 3, 414-420 (1997).

2. Hartmann, G. et al. Oligodeoxynucleotides enhance lipopolysaccharide-stimulated synthesis of tumor necrosis factor: Dependence on phosphorothioate modification and reversal by heparin. Mol. Medicine 2, 429-438 (1996).

3. Webb, A. et al. BCL-2 antisense therapy in patients with non-Hodgkin lymphoma. Lancet 349, 1137-1141 (1997).

\section{Graft persistence in animal models of psoriasis}

To the editor - Creating animal models often provides new and important insights in the pathogenesis of human diseases, and discussing them coram publico is a fruitful means of evaluation. In that regard I appreciate Nature Medicine's coverage of a heated debate' on two new psoriasis models ${ }^{2-6}$. Reviewing the arguments exchanged may be as important as reviewing the original manuscripts and I would like to bring to your attention that the statement by Parker and colleagues on the lack of persistence of human endothelial and epidermal cells in our SCIDhu xenogeneic transplantation model ${ }^{1}$ is misleading. And this is not a minor point, since in particular the persistence of endothelial cells is crucial to studies on leukocyte recirculation which have indeed been performed using our model system (for example, see refs. 7 and 8). Persistence of human endothelia cells has also been shown when the topic to be addressed was psoriasis (eg., ref. 9). 